\title{
Questions to the article: demonstrating the ascendancy of COVID-19 research using acronyms
}

\author{
Shu-Chun Kuo ${ }^{1,2} \cdot$ Tsair-Wei Chien $^{3} \cdot$ Willy Chou ${ }^{4,5}$
}

Received: 29 June 2021 / Accepted: 14 July 2021 / Published online: 5 August 2021

(c) Akadémiai Kiadó, Budapest, Hungary 2021

\begin{abstract}
The article published on 16 May 2021, is well-written and of interest, but remains several questions that are required for clarifications, such as the presentations in Table 1 and Fig. 1 that should be improved further for providing more valuable information to readers. After viewing Table 1 , measuring the strength of quantity $(=0.84)$ referred to the next two counterparts for the top one acronym (e.g., COVID) is demonstrated using the absolute advantage coefficient (AAC). Similarly, Traditional line charts on top-eight acronyms provide us with messages, including (i) DNA and RNA are popular over three decades; (ii) CT, MRI, HIV, SARS, and CoV start in 1972, 1985, 1986, 2003, and 2003, respectively; (iii) the number of COVID substantially surpasses over other seven acronyms in 2020 though the seven acronyms are almost equal in quantity in 2020. We are interested in producing similar Table 1 and Fig. 1 with a video MP4 provided to readers who can click on the link to manipulate the scenarios on their own. We found that the AAC and the traditional line charts on a dashboard make data clear for a better understanding of demonstrating the ascendancy of COVID-19 research using acronyms. The line charts are easily examined on Google Maps.
\end{abstract}

Keywords Google maps · COVID-19 - Absolute advantage coefficient · Line chart · Acronym

Willy Chou

smilewilly@mail.chimei.org.tw

Shu-Chun Kuo

ophkuo@gmail.com

Tsair-Wei Chien

smile@mail.chimei.org.tw

1 Department of Optometry, Chung Hwa University of Medical Technology, Jen-Teh, Tainan City, Taiwan

2 Department of Ophthalmology, Chi-Mei Medical Center, Yong Kang, Tainan, , Taiwan

3 Medical Research Department, Chi-Mei Medical Center, Tainan, Taiwan

4 Department of Physical Medicine and Rehabilitation, Jiali Chi-Mei Hospital, Tainan, Taiwan

5 Chi-Mei Medical Center, 901 Chung Hwa Road, Yung Kung Dist., Tainan 710, Taiwan 
Table 1 Comparison of numbers of acronyms and AACs in two studies

\begin{tabular}{|c|c|c|c|c|c|}
\hline \multirow[t]{3}{*}{ Year } & \multicolumn{5}{|c|}{ Acronyms in titles } \\
\hline & \multicolumn{3}{|c|}{ Barnett and Doubleday(2021) } & \multicolumn{2}{|c|}{ This study } \\
\hline & Acronym & Number & AAC & Number & AAC \\
\hline \multirow[t]{5}{*}{2019} & DNA & 10,873 & \multirow[t]{5}{*}{0.51} & 11,860 & \multirow[t]{5}{*}{0.52} \\
\hline & HIV & 9,485 & & 9,423 & \\
\hline & RNA & 8,636 & & 8,019 & \\
\hline & CT & 4,686 & & 5,590 & \\
\hline & MRI & 4,619 & & 5,687 & \\
\hline \multirow[t]{5}{*}{2020} & COVID & 57,074 & \multirow[t]{5}{*}{0.82} & 64,692 & \multirow[t]{5}{*}{0.84} \\
\hline & SARS & 11,747 & & 12,290 & \\
\hline & DNA & 11,189 & & 12,210 & \\
\hline & $\mathrm{CoV}$ & 10,981 & & 11,817 & \\
\hline & RNA & 10,476 & & 9,482 & \\
\hline
\end{tabular}
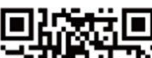

\section{品} $\begin{aligned} \text { Log scale for plots } & \text { Latitude of } \\ \text { covID } & \text { coordinates }\end{aligned}$
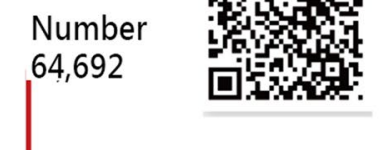
11 82

966

$7 \quad 27$

$2 \quad-32$

Fig. 1 The most popular scientific acronyms in health and medical journals over the years

We read with great interest the study by Barnett and Doubleday on demonstrating the ascendancy of COVID-19 research using acronyms (Barnett \& Doubleday, 2021). However, two major concerns were raised, such as how to (1) measure the strength of quantity referred to the next counterparts and (2) produce the traditional line charts on a dashboard that provides more valuable information to readers.

The absolute advantage coefficient(AAC) (Chang et al., 2020; Chien, 2012; Lee et al., 2020, 2021; Wang et al., 2021; Yang et al., 2021) to report the strength of quantity when compared to the next two counterparts using Eq. (1) and (2): 


$$
\begin{gathered}
\text { Ratio }=\frac{\frac{\gamma_{1}}{\frac{\gamma_{2}}{\gamma_{3}}},}{\mathrm{AAC}=\frac{\text { Ratio }}{(1+\text { Ratio })},}
\end{gathered}
$$

where Ratio is determined by the three consecutive numbers of acronyms(e.g., in 20,220; the top three have the most number of frequency in quantity, denoted by $\gamma_{1}, \gamma_{2}$, and $\gamma_{3}$ in Eq. (1)). The ACC ranged from 0 to 1.0 stands for the strength of quantity in 2020 when compared to the next two acronyms.

We extracted the top eight acronyms involved in the article title shown in Table 1(Barnett \& Doubleday, 2021) and downloaded them from PubMed since 1950. In comparison to the original counts shown in the study (Barnett \& Doubleday, 2021), almost equal counts across the eight acronyms were found in Table 1. The AACs were particularly calculated for the two studies. We can see that the AACs in 2020(>0.80) are substantially greater than those in $2019(\cong 0.50)$. The higher ACC for the top one acronym means stronger in quantity when compared to the next two.

Next, the line charts are shown in Fig. 1. More valuable information is provided to readers, including (i) DNA and RNA are popular over three decades; (ii) CT, MRI, HIV, SARS, and CoV start in 1972, 1985, 1986, 2003, and 2003, respectively; (iii) the number of COVID substantially surpasses over other seven acronyms in 2020 though the seven acronyms are almost equal in quantity in 2020.

We provided a video MP4 with a link at the reference (Chien, 2021a). Readers are invited to scan the QR-code or click on the link (Chien, 2021b) to manipulate the line charts by using the zoom-in and zoom-out functions on a dashboard laid on Google Maps that is unique and modern, and easier to understand the features of frequencies in acronyms than the static line charts shown in the studied (Barnett \& Doubleday, 2020, 2021).

We produced the improved Table 1 and Fig. 1 when compared to the previous study (Barnett \& Doubleday, 2021). The abstract video is present in (Chien, 2021a) for readers who are interested in replicating Table 1 and Fig. 1 on their own in the future.

Authors' Contribution SC developed the study concept and design. WC analyzed and interpreted the data. SC monitored the process of this study and helped in responding to the reviewers' advice and comments. TWC drafted the manuscript, and all authors provided critical revisions for important intellectual content. The study was supervised by TWC. All authors read and approved the final manuscript.

Funding There are no sources of funding to be declared.

Availability of data and material All data used in this study are available (Chien, 2021a).

\section{Declarations}

Competing interests The authors declare that they have no competing interests.

Ethical approval Not applicable.

Consent to participate All data were downloaded from PubMed.

Consent to publish Not applicable. 


\section{References}

Barnett, A., \& Doubleday, Z. (2020). The growth of acronyms in the scientific literature. eLife, 23(9), e60080. https://doi.org/10.7554/eLife.60080 PMID: 32701448; PMCID: PMC7556863.

Barnett, A., Doubleday, Z. (2021). Demonstrating the ascendancy of COVID-19 research using acronyms. Scientometrics, 16, 1-4. https://doi.org/10.1007/s11192-021-04016-8. Epub ahead of print. PMID: 34024958; PMCID: PMC8124093.

Chang, C. S., Yeh, Y. T., Chien, T. W., Lin, J. C. J., Cheng, B. W., \& Lai, F. J. (2020). Using the separation index to identify the most dominant role: A case of application on COVID-19 outbreak. International Journal of Organizational Innovation, 12, 10-20.

Chien, T. W. (2012). Cronbach's alpha with the dimension coefficient to jointly assess a scale's quality. Rasch Meas Trans, 26, 1379.

Chien, T. W. (2021a). Code, data, and results for this article. 2021/6/27 available at http://www.healthup. org.tw/article/course_mb2.asp?repno $=9$

Chien, T. W. (2021b). Figure 1 in this study. 2021/6/28 available at http://www.healthup.org.tw/gps/acron ymskeys.htm

Lee, C. J., Chou, W., Chien, T. W., Yeh, Y. T., \& Jen, T. H. (2020). Using the separation index for identifying the dominant role in an organization: A case of publications in organization innovation. International Journal of Organizational Innovation, 12, 135-145.

Lee, K. W., Chien, T. W., Yeh, Y. T., Chou, W., \& Wang, H. Y. (2021). An online time-to-event dashboard comparing the effective control of COVID-19 among continents using the inflection point on an ogive curve: Observational study. Medicine (baltimore), 100(10), e24749. https://doi.org/10.1097/MD.00000 00000024749 PMID: 33725830; PMCID: PMC7969250.

Wang, L. Y., Chien, T. W., \& Chou, W. (2021). Using the IPcase index with inflection points and the corresponding case numbers to identify the impact hit by COVID-19 in China: An observation study. International Journal of Environental Research and Public Health, 18(4), 1994. https://doi.org/10.3390/ ijerph18041994 PMID: 33670825; PMCID: PMC7923186.

Yang, D. H., Chien, T. W., Yeh, Y. T., Yang, T. Y., Chou, W., \& Lin, J. K. (2021). Using the absolute advantage coefficient (AAC) to measure the strength of damage hit by COVID-19 in India on a growth-share matrix. European Journal Medical Research, 26, 61. 